\title{
Improved Crystal-Growth and Emission Gain-Narrowing of Thiophene/Phenylene Co-oligomers**
}

by Musubu Ichikawa*, Ryota Hibino, Masamitsu Inoue, Takeshi Haritani, Shu Hotta, Toshiki Koyama, Yoshio Taniguchi

[*] Dr. M. Ichikawa, Mr. R. Hibino, Dr. M. Inoue, Mr. T. Haritani, Dr. T. Koyama, Prof. Y. Taniguchi Department of Functional Polymer Science

Faculty of Textile Science and Technology

Shinshu University

Tokita 3-15-1, Ueda, Nagano 386-8567 (Japan)

E-mail: musubu@giptc.shinshu-u.ac.jp

Dr. S. Hotta

Institute of Research and Innovation

Kashiwa Laboratory

Photonics and Materials Research Department

1201 Takada, Kashiwa, Chiba 277-0861 (Japan)

[**]This work was supported by the New Energy and Industrial Technology Development Organization (NEDO) for Organic Materials Technology for a Solid-State Injection Laser theme. This work was also supported by a Grant-in-Aid for COE Research (10CE2003) from the Japanese Ministry of Education, Culture, Sports, Science, and Technology. 
Unique optoelectronic features have been demonstrated using molecular semiconductors in (poly)crystalline phases. Examples include lasing and amplified spontaneous emission (ASE) from acene-, phenylene-, or thiophene-based materials that are photoexcited or charge-injected through electrodes on suitably constructed devices. Accordingly, the crystals of high quality are increasingly needed to achieve high optoelectronic performance.

To this end, various crystal-growth techniques have been proposed and developed to date. As a typical example, single crystals such as anthracene and naphthalene were made via well-established techniques, e.g. the zone-melting method and the Bridgman method. More recently, Kloc et al. proposed a crystal-growth method in the vapor phase under a considerably high pressure of environmental gas (such as argon, helium, and hydrogen). ${ }^{[1,2]}$ That method made accessible large single crystals of the molecular materials. For instance the authors made crystals of $\alpha$-sexithiophene of $\sim 1 \mathrm{~cm}$ in size both in closed and open systems.

On the basis of the above technique, we further tried to improve the high-pressure crystal-growth in the closed system. The point is that since the crystals are grown under a quasi-equilibrium state, the growth can desirably be tuned by e.g. carefully regulating the growth temperature and controlling the gas pressure inside the apparatus. After elaborating some pieces of apparatus of crystal-growth glassware, we found out that the apparatus made of an axially symmetric glass tube (such as a test tube) have given the best results. Thus, we have been successful in preparing crystals of several $\mathrm{mm}$ to $\sim 1 \mathrm{~cm}$ in size and of optically high transparency.

Meanwhile, we have already reported the ASE from the as-synthesized and melt-recrystallized materials regarding several molecular semiconductors, including oligophenylenes and thiophene/phenylene co-oligomers. ${ }^{[3,4]}$ The family of these oligomer materials is characterized by the well-defined molecular axes and exhibits various interesting electric transport and light emission features on the basis of the disposition of their axes in thin films and crystals. ${ }^{[5-8]}$ In particular, the 
thiophene/phenylene co-oligomers have a variety of extension of $\pi$-conjugation along the backbone. The conjugation extension can be tuned by changing the total number of the thiophenes and phenylenes and their mutual arrangement in the molecules. We applied the aforementioned crystal-growth method to some of these co-oligomers and the resulting crystals definitively caused the ASE. In this communication we describe the improved crystal-growth technique and interesting aspects of the self-waveguided ASE.

Scheme 1 shows the structural formulae and abbreviated chemical notations of the materials. Scheme 2 depicts cross sectional and side views of the growth apparatus. The apparatus is placed on a uniformly heated hot plate (of a size large enough compared to the growth apparatus) with its cylinder axis parallel to the hot plate surface. In this closed apparatus material loss is minimized. The temperature of the individual cylinder walls is the lowest at the top of the cylinder and the highest at the bottom of the cylinder, with which the solid sample is close contact. The growth apparatus is wrapped by high thermal conductivity materials such as aluminum foils and copper blocks so that the temperatures of the walls including the top and the bottom can be regulated as desired. The presence of an environmental gas (such as argon) inside the apparatus encourages the convective transfer of the material. The initial pressure of the charged gas was suitably regulated such that its pressure reaches the atmospheric pressure at the growth temperature. On this condition, matters are slowly transported from the bottom to the top of the cylinder by the convection. The mean free path of vaporized molecules is estimated to be a few nanometers at a growth temperature (500 $700 \mathrm{~K}$ ). Neither diffusion nor molecular flow takes place even at the highest growth temperatures.

We confirmed such convective material transport through direct observation of fine crystals as schematically depicted in Scheme 2(b). The deposition of the transported matters starts at the top of the cylinder and the crystal growth continues overnight. To obtain high quality single crystals, regulating the temperature and the growth rate is essential. High growth temperatures provide enough energies for the 
adsorbed molecules to desorb and reorient. High pressures inside the apparatus assist the slow crystal growth at high temperatures. These thermodynamic conditions ensure the generation of defect-free large crystals (several $\mathrm{mm}$ to $\sim 1 \mathrm{~cm}$ in size). Thicknesses of the crystals obtained from the same growth batch were varied from about $1 \mu \mathrm{m}$ to 20 $\mu \mathrm{m}$. Crystals of about 3 to $5 \mu \mathrm{m}$ in thickness were selected for the subsequent experiments. Growth conditions for the individual materials are summarized in Table 1.

Figure 1 shows the optical microscope and fluorescence microscope images of a plate crystal of BP2T. As shown in Figure 1(a), the BP2T crystal is a smooth-surfaced single platelet of about $3 \times 2.5 \mathrm{~mm}^{2}$ in size. Since Figure 1(a) shows interference fringes between the sample stage surface and the crystal surface faced to the sample stage, the surface smoothness is confirmed. Notice that interference fringes are disappeared when the crystal was placed on a mat surfaced stage. Figure 1(b) shows another optical micrograph, in which orange emission from the BP2T crystal is clearly visible when it is excited by UV lights from a handy Hg lamp. As evidenced in this figure, the orange fluorescence is noticeable only at edges of the crystal without emissions from the inside at all. This implies that the self-waveguided fluorescent emission is occurring from the sample crystal whose morphology is not polycrystalline. The micrograph observations of all the crystals (i.e. BP1T, BP2T, BC4, and AC5) under crossed Nicols configuration of the polarizer and analyzer indicate that bright and dark field images are switched at each $45^{\circ}$-rotation.

Figure 2(a) shows laser induced self-waveguided light emission spectra from a BP1T single crystal as a function of the fluence of the incident laser pulse. It should be noted here that the sample crystal was thin enough for a relatively large amount of the incident light to reach and excite the parts near the bottom crystal face because the absorption coefficient of BP1T crystal at $337 \mathrm{~nm}$ was about $0.05 \mu \mathrm{m}^{-1}$. This small absorption coefficient will be associated with the fact that the crystal of BP1T is $a b$-platelet (to which the molecular long axis is vertically disposed). ${ }^{[9]}$ The laser induced self-waveguided emission spectrum at the incident excitation fluence of $5 \mu \mathrm{J} / \mathrm{cm}^{2}$, 
which exhibit a broad feature with relatively fine modes superposed on it, was similar to emission spectra under weaker excitation by a monochromatic Xe lamp. These fine modes can be assigned to the vibronic structures that are frequently observed in solid-phase molecules with a well-defined molecular symmetry. ${ }^{[4]}$ As shown in this figure, at higher excitation, only the broad photoluminescence band at $498 \mathrm{~nm}$ is progressively narrowed down to a line width of approximately $3 \mathrm{~nm}$ full width at half maximum (FWHM) and its maximum is slightly blue-shifted to $495 \mathrm{~nm}$. Figure 3 represents the self-waveguided light emission peak intensity and their FWHMs as a function of the incident laser fluence. When the incident laser fluence is increased, the emission peak intensities exponentially grow, accompanied by the narrowing as shown in the figure. Therefore, we conclude that the narrowing is caused by gain-narrowing, namely, ASE. ${ }^{[10-12]}$ Reflecting the fact that the crystal of BP1T is $a b$-platelet, ${ }^{[9]}$ the transverse magnetic (TM) modes are dominant with no correlation in excitation laser fluence. This TM dominance is also the case with all the other samples investigated (see Table 1).

As another example of the induced self-waveguided light emission, related spectra from an AC5 single crystal are represented in Figure 2(b). A broad emission spectrum peaked at $516 \mathrm{~nm}$ is progressively narrowed with increased excitation fluences and replaced with a sharp line peaked at $515 \mathrm{~nm}$ with the FWHM of approximately $3.4 \mathrm{~nm}$. Thus, the self-waveguided ASE is again evident. Table 2 summarizes the results of laser induced self-waveguided ASE characteristics.

The threshold for the self-waveguided ASE of the BP1T single crystal was estimated to be $27 \mu \mathrm{J} / \mathrm{cm}^{2}$ as presented in Figure 3. This was defined from the incident laser fluence as the halfway point from fluorescence to the completely narrowed ASE. This threshold fluence can be converted to the threshold peak power density of 54 $\mathrm{kW} / \mathrm{cm}^{2}$ by dividing the said fluence by the pulse duration (500 ps) of the excitation laser. This threshold is extremely low compared with those for as-synthesized crystals (ca. $500 \mu \mathrm{J} / \mathrm{cm}^{2}$ ) and melt-recrystallized solids $\left(150 \mu \mathrm{J} / \mathrm{cm}^{2}\right)$ reported previously 
regarding the same material. ${ }^{[3,4]}$ The low threshold demonstrated in the present studies reflects the high quality and transparency encompassing the whole crystal and is a consequence of an efficient confinement of self-waveguided lights. Correspondingly, the gain length is expected to be two or three orders of magnitude longer at the same incident laser fluence than that for the as-synthesized small crystals. ${ }^{[13]}$ Note that the as-synthesized crystals are of several tens of $\mu \mathrm{m}$ in size. The following is additionally worth mentioning: In both the cases of the as-synthesized and melt-recrystallized materials of BP1T and AC5, we pointed out previously that whereas two lines were gain-narrowed at once in some cases, in other cases either of those two was preferentially gain-narrowed according to the individual measurements. ${ }^{[3,4]}$ We sought the origin from the difference either in size or in texture of the crystals or melt-recrystallized materials. In this context only one line is reproducibly gain-narrowed at the same position in the present studies. This is probably the case with the single crystals of large size that also produces the large gain length.

In conclusion, we described the improved crystal-growth technique for organic semiconductor materials and interesting aspects of the ASE. The improved technique provided large plate single crystals of several $\mathrm{mm}$ to $\sim 1 \mathrm{~cm}$ in size, and the prepared single crystals exhibited self-waveguided ASE. The observed ASEs with a relatively small threshold result from excellent optical properties of the crystals (e.g. a high transparency, low light-scattering, an efficient confinement of self-waveguided lights, etc.).

\section{Experimental}

The synthesis and purification methods of the materials can be seen elsewhere. ${ }^{[14]}$ The procedures of the crystal growth are as follows: (i) The material was put inside a glass tube (10 $\mathrm{cm}$ in length and $3 \mathrm{~cm}$ in diameter) and the tube was molten and narrowed near the open edge. The tube was evacuated, replaced with dry argon, and then sealed near that edge. The pressure of the argon gas was regulated such that the 
temperature during the crystal growth could be $\sim 1 \mathrm{~atm}$. (ii) This apparatus was placed on a hot plate and the temperatures of the plate were appropriately adjusted according to the difference in the materials. The temperatures were monitored on the hot plate close to the position at which the apparatus was in contact with the plate, and monitored on the top of the apparatus, too. Normally, the temperature was held just below the melting temperature of the material. (iii) The apparatus was covered with an aluminum foil and the crystals typically of several mm were generated in $\sim 20 \mathrm{~h}$.

The optical and fluorescence microscope observations were performed using a digital microscope (Keyence, VH-8000), and the polarized micrograph was taken with OPTIPHOT-POL (Nikon). An $\mathrm{N}_{2}$-gas laser that generated a light pulse of 500 -ps-duration at $337 \mathrm{~nm}$ at a repetition rate of $10 \mathrm{~Hz}$ was used as an excitation light source for the gain-narrowing experiment. The excitation laser light was normally incident to the crystal face, focusing on about $1 \times 3 \mathrm{~mm}^{2}$ in size at that face. Lights emitted from the inner parts of the crystal traveled along the crystal faces and were detected at an end-edge of the crystal. The lights were collected with an optical fiber bundle through a UV-cut filter and analyzed by a spectrograph (Solar TII, MS7504) equipped with a charge-coupled device camera (Andor, DV420-OE). To determine which propagation mode(s) of the self-waveguided emission lights is dominant, we have measured emissions polarized either parallel or perpendicular to the crystal surfaces. 
References

[1] C. Kloc, P. G. Simpkins, T. Siegrist, R. A. Laudise, J. Cryst. Growth, 1997, 182, 416.

[2] R. A. Laudise, C. Kloc, P. G. Simpkins, T. Siegrist, J. Cryst. Growth, 1998, 187, 449.

[3] R. Hibino, M. Nagawa, S. Hotta, M. Ichikawa, T. Koyama, Y. Taniguchi, Adv. Mater., 2002, 14, 119.

[4] M. Nagawa, R. Hibino, S. Hotta, H. Yanagi, M. Ichikawa, T. Koyama, Y. Taniguchi, Appl. Phys. Lett., 2002, 80, 544.

[5] Y. Yoshida, N. Tanigaki, K. Yase, S. Hotta, Adv. Mater., 2000, 12, 1587.

[6] H. Yanagi, T. Morikawa, S. Hotta, K. Yase, Adv. Mater., 2001, 13, 313.

[7] X. M. Hong, H. E. Katz, A. J. Lovinger, B.-C. Wang, K. Raghavachari, Chem. Mater., 2001, 13, 4686.

[8] M. Ichikawa, H. Yanagi, Y. Shimizu, S. Hotta, N. Suganuma, T. Koyama, Y. Taniguchi, Adv. Mater., 2002, 14, 1272.

[9] S. Hotta, M. Goto, Adv. Mater., 2002, 14, 498.

[10] M. D. McGehee, A. J. Heeger, Adv. Mater., 2000, 12, 1655.

[11] D. Fichou, S. Delysse, J.-M. Nunzi, Adv. Mater., 1997, 9, 1178.

[12] G. Horowitz, P. Valat, F. Garnier, F. Kouki, V. Wintgens, Opt. Mater., 1998, 9, 46.

[13] L. W. Casperson, A. Yariv, IEEE J Quantum Elect, 1972, 8, 80.

[14] S. Hotta, H. Kimura, S. A. Lee, T. Tamaki, J. Heterocyclic Chem., 2000, 37, 281. 
Schemes, Figures, and Tables

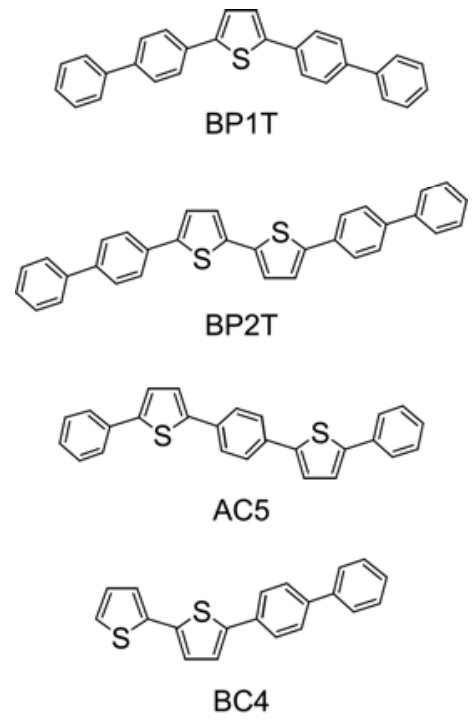

Scheme $1 \quad$ Structural formulae and abbreviated notations of the materials.

(a) Sectional view

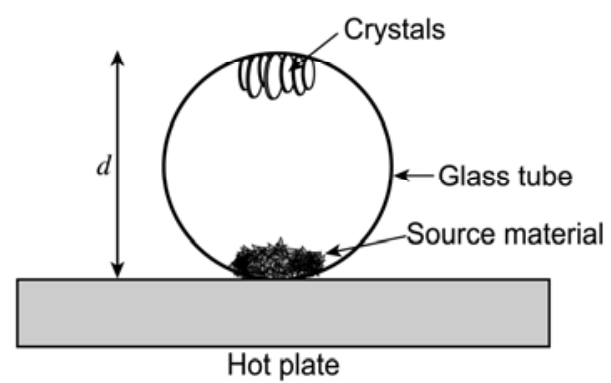

(b) Side view

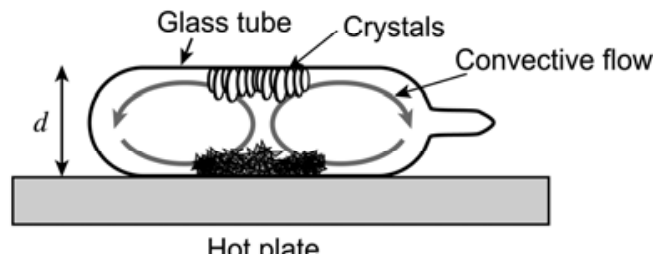

Hot plate

Scheme 2 Cross sectional view (a) and side view (b) of the growth apparatus and schematic representation of the crystal-growth processes. $d$ is a diameter of the growth apparatus. 

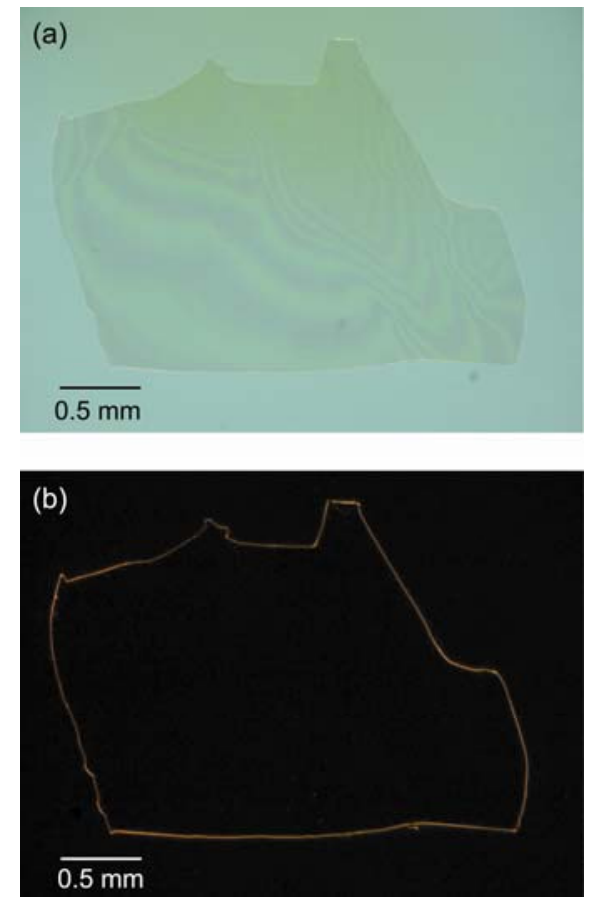

Figure 1

Optical (a) and fluorescence (b) microscope images of the BP2T crystal. 

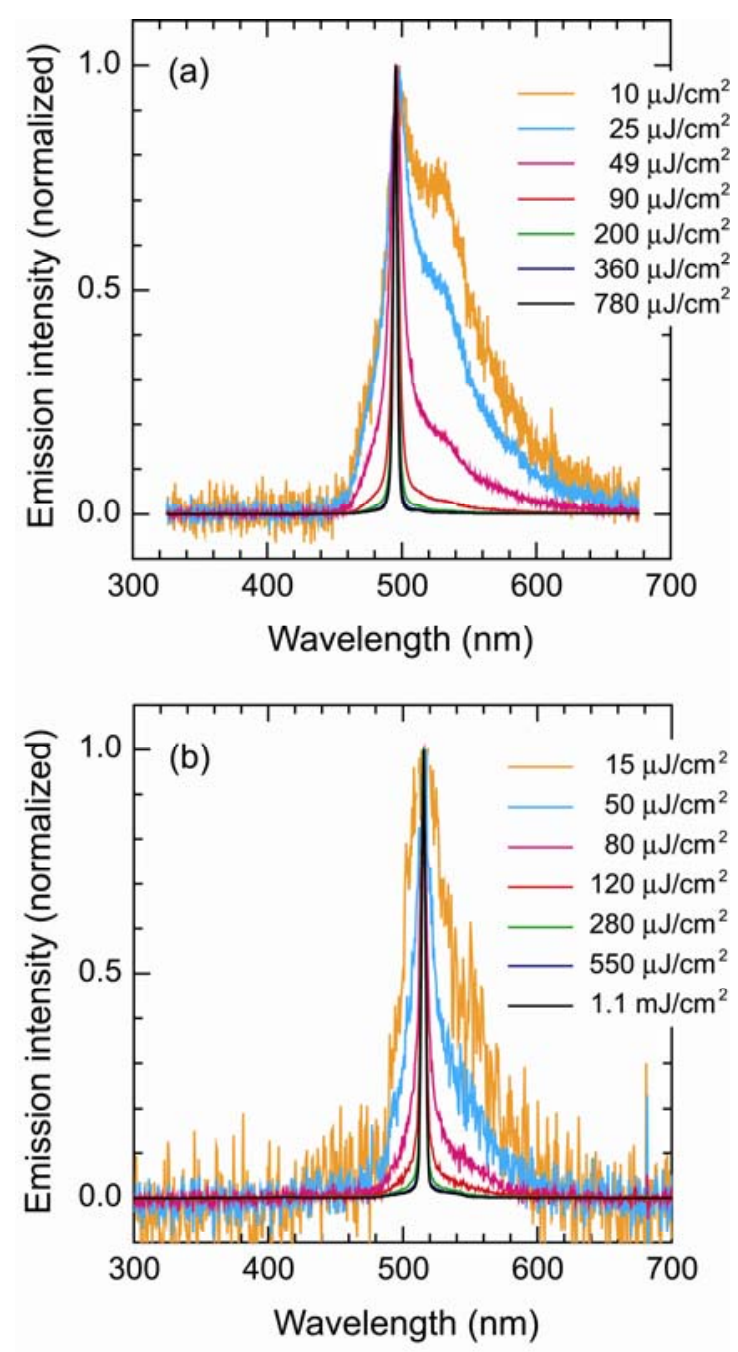

Figure 2 Laser induced self-waveguided light emission spectra from BP1T (a) and AC5 (b) single crystals as a function of the incident laser fluence. 


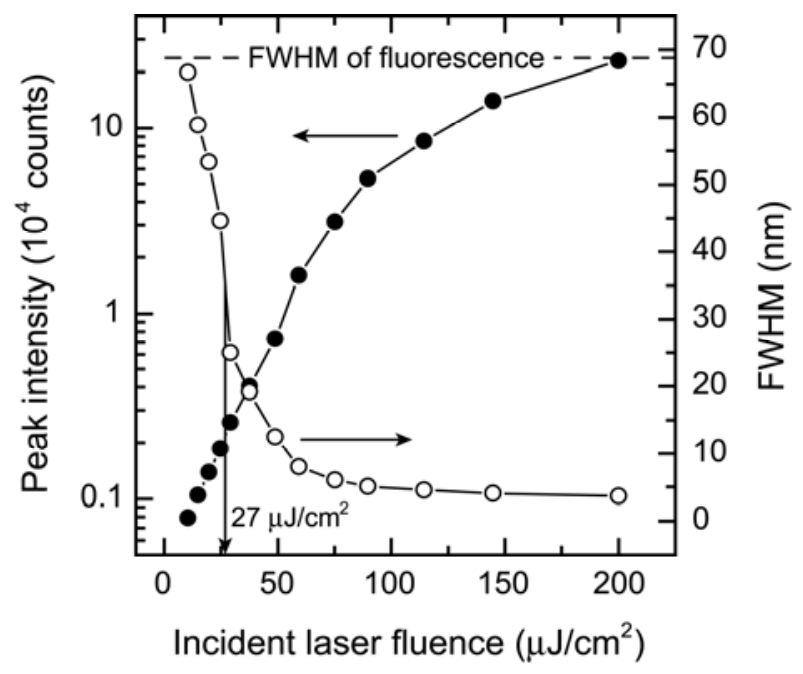

Figure 3 Self-waveguided light emission peak intensity for the gain-narrowed line (around $495 \mathrm{~nm}$ ) and their FWHMs of a BP1T single crystal as a function of the incident laser fluence.

Table $1 \quad$ Growth conditions for the materials.

\begin{tabular}{ccccc} 
Material & $\begin{array}{c}\text { Melting point } \\
(\mathrm{K})\end{array}$ & $\mathrm{T}^{\mathrm{a})}(\mathrm{K})$ & $\mathrm{T}^{\mathrm{b})}(\mathrm{K})$ & $\begin{array}{c}\text { Pressure }^{\mathrm{c})} \\
(\mathrm{kPa})\end{array}$ \\
\hline \hline BP1T & $602^{\mathrm{d})}$ & 593 & 559 & 50 \\
BP2T & $631^{\mathrm{d})}$ & 597 & 547 & 50 \\
BC4 & $499^{\mathrm{d})}$ & 488 & 447 & 60 \\
AC5 & $577^{\mathrm{d})}$ & 543 & 505 & 60 \\
\hline
\end{tabular}

a) Temperature of the growth-cylinder at bottom

b) Temperature of the growth-cylinder at top

${ }^{c)}$ Charging pressure of gwoth-gas ( $\mathrm{Ar}$ ) at room temperature

${ }^{d)}$ Ref. 14 
Table 2 Positions, FWHMs, TM/TE ratios, and threshold excitation fluences of the gain-narrowed lines for single crystals of various materials.

\begin{tabular}{cccccc} 
Material & $\begin{array}{c}\text { Wavelength } \\
(\mathrm{nm})\end{array}$ & $\begin{array}{c}\text { FWHM } \\
(\mathrm{nm})\end{array}$ & $\begin{array}{c}\text { TM/TE } \\
\left(\mathrm{cm}^{-1}\right)\end{array}$ & $\begin{array}{c}\text { Threshold } \\
\text { ratio }\end{array}$ & $\begin{array}{c}\text { a) } \\
\left(\mu / \mathrm{cm}^{2}\right)\end{array}$ \\
\hline \hline BP1T & 495 & 3.2 & 130 & 2.0 & 27 \\
BP2T & 565 & 5.2 & 169 & 3.3 & 355 \\
BC4 & 499 & 5.0 & 189 & 7.8 & 50 \\
AC5 & 515 & 3.4 & 128 & 2.0 & 42 \\
\hline
\end{tabular}

a) Intensity ratio of two light-propagation modes (transverse magnetic modes and transverse electric modes) at the excitation power density of $1 \mathrm{~mJ} / \mathrm{cm}^{2}$ 